\title{
Evaluation of Polymer Modification in Asphalt Mixtures through Digital Image Correlation and Performance Space Diagrams
}

\author{
Brian Hill \\ Graduate Research Assistant \\ Department of Civil \& Environmental Engineering, \\ University of Illinois at Urbana-Champaign \\ 205 N. Mathews MC 250, Urbana, IL 61801 \\ Email: bchill2@illinois.edu \\ William G. Buttlar \\ Professor \\ Department of Civil and Environmental Engineering \\ University of Illinois at Urbana-Champaign \\ 205 N Mathews Ave., MC-250, Urbana, IL 61801 \\ E-mail: buttlar@uiuc.edu
}

\begin{abstract}
Asphalt polymer modification has yielded improved mixture performance. Little research has been conducted to determine how SBS polymer affects mixture performance in interaction plots. Furthermore, research to date has not used digital image correlation to measure the fracture process zone (FPZ) size and its compression with crack propagation. Mixtures containing five distinct asphalt binders were tested at high and low temperatures. Findings demonstrated that the PG 70-28 mixture shifted optimally in terms of high and low temperature properties. It was also found that polymer addition led to increased FPZ size and decreased FPZ compression with propagation.
\end{abstract}

\section{Introduction}

Forensic research of cracking and rutting distresses in the field often demonstrate the superior performance of polymer-modified asphalt compared to unmodified asphalt mixtures. Von Quintus and Mallela (2005) and von Quintus et al. (2007) found polymer modification led to increased service life of up to 10 years in Colorado and throughout the United States based on field investigations. In addition, Timm et al. (2013) determined that polymer-modified asphalt yielded similar or improved performance compared to companion unmodified asphalt field sections. Field studies such as these provide impetus for laboratory research to understand how polymer positively affects asphalt binder and mixture properties at the macroscale (greater than centimeter scale) and microscale (sub-millimeter scale).

Laboratory investigations have shown that polymer modification leads to improved high and low temperature properties. At high temperatures, Woo et al. (2007) noted improvements in shear stiffness in unaged binder via the dynamic shear rheometer test for polymer modified mixtures, which is associated with increased rutting resistance. Further evidence of superior rutting performance was found in laboratory binder studies with multiple stress creep and recovery tests 
completed by Vahidi et al. (2014) and Yoo et al. (2015). Finally, low temperature binder performance indicators through measurements of fracture energy density by Yoo et al. (2015) showed that polymer addition can lead to improved low temperature cracking resistance.

Laboratory research at the mixture macroscale level has produced analogous results to the asphalt binder investigations. Mohammad et al. (2011), Vahidi et al. (2013), and Timm et al. (2014) found improved high temperature performance in polymer-modified mixtures through loaded wheel tests such as the Hamburg wheel tracking test. Furthermore, intermediate and low temperature cracking studies such as Birgisson et al. (2011), Montepara et al. (2011A and B), Elseifi et al. (2012), and Braham et al. (2013) illustrated improved mixture performance resulting from polymer modification.

One method of evaluating macroscale-level improvements to mixtures resulting from polymer modification is to employ interaction plots to simultaneously evaluate high and low temperature mixture properties. This type of plot provides the user with a graphical means to understand shifts in rutting and cracking performance caused by changes in mixture ingredients, mixture composition and/or mixture volumetrics. Zhou et al. (2013) and Cooper III et al. (2014) used this concept to evaluate recycled asphalt sources, binder grades, etc. in terms of rutting and fatigue or reflective cracking. Buttlar et al. (2016) used this plot to evaluate field mixtures, binder grades, aggregate types, recycled asphalt pavement, recycled asphalt shingles, and recycled engine oil bottoms in terms of rutting and low temperature cracking resistance. Ozer $e t$ al. (2016) also demonstrated the use of interaction plots to evaluate asphalt mixtures. However, none of these studies examined how incremental dosage rates of SBS effect mixture behavior in terms of shifts in a high-low temperature interaction space.

Mixture microscale low temperature properties have significance in terms of understanding polymer modification effects on fracture. Research by Birgisson et al. (2011) and Montepara et al. (2011A and B) considered strain amplification/localization at cracks through qualitative assessment of DIC measurements. In Portland cement concrete research, Bazant et al. (1990) and Das et al. (2015) measured the fracture process zone (FPZ) maximum length through analytical and DIC image analysis, respectively. However, little to no research has been completed to understand the effects of polymer modification in asphalt via a measure of the FPZ. Furthermore, little research has been conducted to consider how the FPZ changes in asphalt with and without modifiers during the period of testing. Measurements of the FPZ may provide meaningful information regarding strain dispersion due to polymer addition in asphalt mixtures thereby explaining how polymer modifiers improve low temperature fracture properties. Thus, the current research study will focus on macroscale interaction plots as well as measures of microscale low temperature mixture properties to further examine polymer modification effects in asphalt mixtures.

\section{Objectives}

The goal of the current study aimed to evaluate the effects of incremental dosage rates of SBS polymer on high and low temperature mixture performance properties at multiple length scales. First, Hamburg wheel tracking and DC(T) and SCB fracture tests were conducted to evaluate the macroscale properties of the polymer-modified and unmodified asphalt mixtures at high and low 
temperatures, respectively. Second, the Hamburg-DC(T) diagram was employed to evaluate how the SBS polymer content shifted performance. Third, the microscale properties of the asphalt mixtures at low temperatures using both $\mathrm{SCB}$ and $\mathrm{DC}(\mathrm{T})$ geometries were examined by measuring the size and dissipation of the fracture process zone using DIC.

\section{Methods and Materials}

\subsection{Testing Materials}

This studied used a $9.5 \mathrm{~mm}$ nominal maximum aggregate size (NMAS) mixture to evaluate the incremental dosage rate effects of SBS polymer. Dosage rates of SBS polymer were $1.5 \%$ for the PG 70-22 and PG 70-28 binders and 3.0\% for the PG 76-22 binder. In addition, two unmodified mixtures containing PG 64-22 (control mixture) and PG 58-28 were used for comparisons between modified and unmodified mixtures. SBS is a common polymer modifier found in asphalt cements. This polymer, which is also used in the shoe sole and tire industries, is a block copolymer consisting of three units. The first unit, polystyrene, is a tough hard plastic providing added stiffness to the asphalt cement. The second unit, polybutadiene, is a rubbery component providing added elastic recovery to the asphalt cement. In addition, SBS is a thermoplastic elastomeric polymer. Asphalt viscosity measurements were completed at $130^{\circ} \mathrm{C}$ using a Brookfield Rotational Viscometer under the ASTM D2171 standard. The viscosity results for the PG 64-22, 58-28, 70-22, 70-28, and 76-22 binders were $0.6,0.4,0.9,0.8$, and 9.0 Pa-s, respectively.

The mixtures contained crushed limestone and natural sand fine aggregate and crushed gravel coarse aggregate. Mixtures designed in the current study followed AASHTO M323 to meet Superpave volumetric requirements. The unmodified mixtures were mixed and compacted at $150^{\circ} \mathrm{C}$ while the modified mixtures were mixed at $170^{\circ} \mathrm{C}$ and compacted at $150^{\circ} \mathrm{C}$. These temperatures were chosen in order to comply with the mixture design asphalt binder viscosity recommendations according to Roberts et al. (1996). All mixtures and subsequent performance test specimens were aged for approximately 2 hours prior to compaction for short term oven aging. Additionally, all mixtures were stirred at approximately 1 hour after introduction to the short term aging oven to avoid aging gradients with the sample. The mixture gradations were chosen such that volumetric properties such as the voids in the mineral aggregate (VMA), voids filled with asphalt (VFA), and percent effective binder $\left(\mathrm{P}_{\text {be }}\right)$ were approximately equal to avoid additional variables affecting performance in the $\mathrm{DC}(\mathrm{T})$ and Hamburg tests. The volumetric properties for the virgin aggregate and recycled material mixtures are provided in Table $\mathbf{1 .}$ Mixture DesignTable 1. The recycled binder contents in percentage form were provided in terms of the asphalt binder replacement ratio (ABR). 
Table 1. Mixture Design Summary

\begin{tabular}{|c|c|}
\hline Total Asphalt Content (\%) & 6.6 \\
\hline ABR (\%) & 0.0 \\
\hline Air Voids (\%) & 4.0 \\
\hline VMA (\%) & 15.2 \\
\hline VFA (\%) & 74.0 \\
\hline Effective Asphalt Content (\%) & 4.9 \\
\hline Dust/Effective AC & 1.1 \\
\hline
\end{tabular}

\subsection{High and Low Temperature Tests}

The Hamburg wheel tracking test (HWT) was used to evaluate the high temperature macroscale properties of the asphalt mixtures investigated in this study. Following AASHTO T-324 (2014), the HWT was used to measure mixture permanent deformation via external linear variable transducers (LVDT) in a water-immersed state at $50^{\circ} \mathrm{C}$ with a $702 \mathrm{~N}$ loading applied through a rolling steel wheel. Asphalt mixture gyratory-compacted specimens, $130 \mathrm{~mm}$ in height, were cut in half and sawn along one edge to produce a flat face to produce a geometry suitable for the HWT (using the cylindrical geometry option). The heights of the two sides of each gyratory specimen were adjusted to reach equal heights to avoid dynamic loading. All Hamburg tests were conducted until either 20,000 passes was reached or $20.0 \mathrm{~mm}$ of rut depth was induced.

Several states specify the HWT to control asphalt pavement rutting. The Illinois Department of Transportation (IDOT) has three specification levels, where the minimum number of wheel passes prior to reaching an average rut depth of $12.5 \mathrm{~mm}$ is specified. When a PG 58-28 asphalt binder is specified, a minimum of 5,000 wheel passes is specified. When a PG 64-22 asphalt binder is specified, a minimum of 7,500 wheel passes must be reached prior to reaching a 12.5 $\mathrm{mm}$ rut depth. Finally, if mixtures are placed in the Chicago area, they must reach 20,000 passes in the HWT prior to reaching a $12.5 \mathrm{~mm}$ rut depth. The current study used the 7,500 wheel pass requirement for the Hamburg-DC(T) diagram, as the control (unmodified) mixture contained PG 64-22 asphalt binder.

$\mathrm{DC}(\mathrm{T})$ and SCB fracture test geometries were employed to measure the low temperature macroscale properties of the asphalt mixtures. Both geometries measure the crack propagation potential for asphalt mixtures in tension (Mode I fracture). Marasteanu et al. $(2007,2012)$ and Wagoner et al. (2005) examined multiple fracture geometries, including the DC(T) and SCB, in Federal Highway Administration (FHWA) and National Science Foundation studies, respectively. Specific details regarding the geometries can be found in Marasteanu et al. (2007). The FHWA study created three minimum DC(T) mixture fracture energy levels based on the amount of traffic (project criticality) at the placement location to add a layer of risk avoidance. The thresholds for low, medium, and high traffic asphalt pavement mixtures were set at 400, 460 , and $690 \mathrm{~J} / \mathrm{m}^{2}$, respectively, for short-term oven aged ( 2 hour oven aged) samples. 


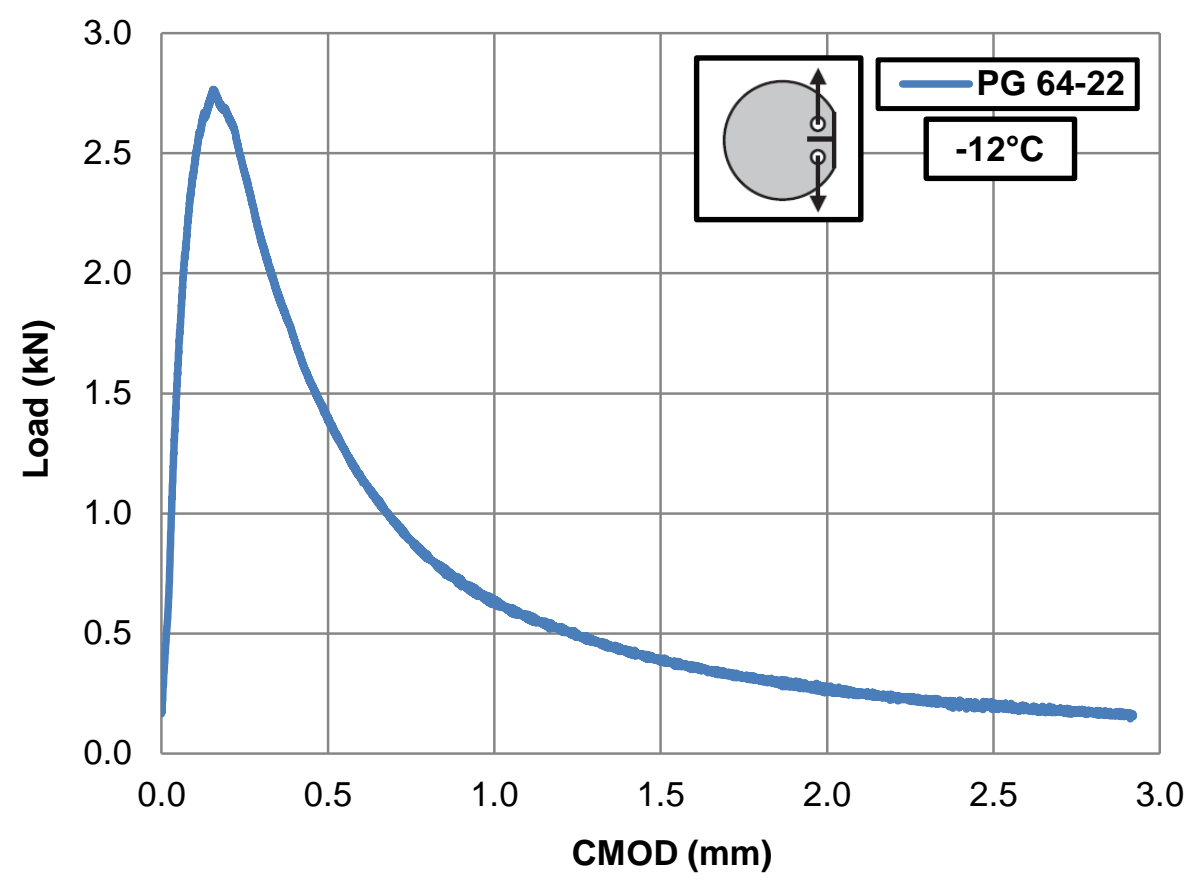

Fig. 1. PG 64-22 Mixture DC(T) Load-CMOD Response

The DC(T) and SCB tests evaluated fracture energy by measuring the area under the load-crack mouth opening displacement (CMOD) gauge curve, shown in Fig. 1, and normalizing it by the fractured surface area as shown in the following equations:

$$
\begin{aligned}
& A=\int_{0}^{\delta_{\max }} P(\delta) d \delta \\
& G_{f}=\frac{A}{b L}
\end{aligned}
$$

where $G_{f}, \delta, \delta_{\max }, A, b, L$, and $P(\delta)$ are the CMOD fracture energy, CMOD, maximum CMOD, area under the load-CMOD curve, fracture area width, fracture area length, and the load at a specific CMOD value, respectively. Further details regarding the DC(T) test are found in ASTM D-7313 (2014).

The current study tested all specimens at $-12^{\circ} \mathrm{C}$ which corresponded to the ASTM recommendation for asphalt mixtures placed in Illinois with a PG 64-22 binder. The fracture specimens were held at $-12^{\circ} \mathrm{C}$ for a minimum of 2 hours prior to testing. All DC(T) and SCB tests were completed at a CMOD opening rate of $1.0 \mathrm{~mm} / \mathrm{min}$ and $0.5 \mathrm{~mm} / \mathrm{min}$, respectively. Testing equipment included an Instron 8500 servo-hydraulic load frame with a $10 \mathrm{kN}$ load cell and an Epsilon 3541 CMOD gauge. All equipment met the ASTM D7313 specifications for testing equipment resolution. A minimum of 2 replicates per mixture were tested to evaluate the average fracture properties. Samples were compacted to approximately $7.0 \%$ air voids in accordance with the ASTM standard. 


\subsection{Hamburg-DC(T) Diagram}

The Hamburg-DC(T) diagram provides a graphical tool to compare mixtures and identify the effects caused by changing various mixture properties. As stated previously, Buttlar et al. (2016) examined the use of this tool to consider effects such as recycled asphalt pavement and shingles, aggregate type, and 3.0\% SBS polymer addition. The goal of any potential asphalt mixture is to lie in the upper right (white) region and avoid the other three corners of diagram. Mixtures in the upper left region lack fracture resistance which would likely lead to poor cracking performance. Mixtures in the lower regions lack rutting resistance, leading to mixture instability. An example of the Hamburg-DC(T) diagram with the use of recycled asphalt shingles is shown below in Fig. 2. The points shown in the example include: the control mixture (PG 64-22), a second, softer unmodified mixture (PG 58-28), and experimental mixtures (2.5 and 5.0\% RAS mixtures). The Hamburg-DC(T) diagram demonstrates that the use of a softer asphalt grade led to an unstable PG 58-28 mixture (greater than $12.5 \mathrm{~mm}$ rut depth reached in the HWT). However, by adding RAS to the PG 58-28 binder, mixtures with acceptable fracture and rutting resistance resulted. Furthermore, the RAS mixtures created performed better overall in the Hamburg-DC(T) space, as their corresponding data points were shifted towards the upper-right region of the plot, as compared to the PG64-22 and PG 58-28 mixtures containing only original (virgin) materials.

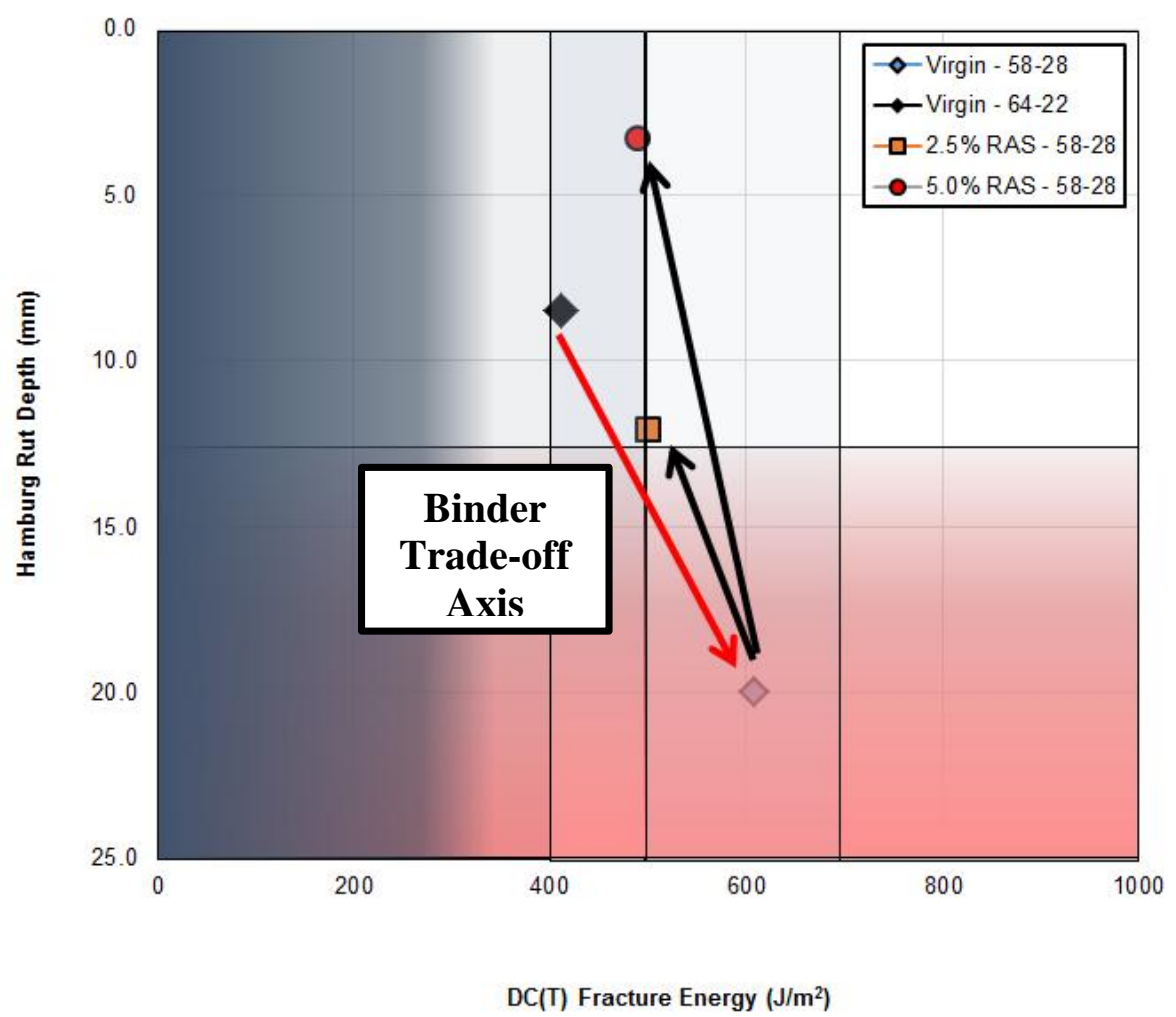

Fig. 2. RAS Mix Designs Illustrated in the Hamburg-DC(T) Space. (Buttlar et al. (2016)) 


\subsection{DIC Application}

The low temperature microscale properties of the asphalt mixtures were evaluated using DIC strain fields for both DC(T) and SCB fracture tests. Several DIC matching algorithms, which minimize measured error and approximate displacement and strain fields, have been developed to evaluate material continuum behavior (Abanto-Bueno et al. (2002) and Carroll et al. (2013)). However, due to the discrete crack propagation which occurs during the fracture process in asphalt concrete, the Poissant and Barthelat (2011) DIC matching algorithm was used. This method employs a Newton-Raphson optimization scheme. When a discontinuity/crack propagates through a particular subset, the image subset is split into master and slave subsets. Poissant and Barthelat (2011) used linear subset shape functions to evaluate DIC-measured deformations, as shown in the following equations:

$$
\begin{aligned}
& u(i, j)=u+\frac{\partial u}{\partial x} i+\frac{\partial u}{\partial y} j \\
& v(i, j)=v+\frac{\partial v}{\partial x} i+\frac{\partial v}{\partial y} j
\end{aligned}
$$

where $u$, and $v$ are the horizontal displacement and vertical displacement for the center of a subset. The best parameter match, $q=\left(u, v, \frac{\partial u}{\partial x}, \frac{\partial u}{\partial y}, \frac{\partial v}{\partial x}, \frac{\partial v}{\partial y}\right)$, minimizes the least-squares correlation coefficient.

The DIC set-up used in this study consisted of a Stingray F-201C CCD camera, a Tamron 50mm lens, and multiple LED light sources. A schematic of the set-up at the University of Illinois laboratory is shown in Fig. 3. The Stingray F-201C camera is a 2 megapixel (MP) camera which captures images at a rate of 5 images/sec using a LabVIEW developed program and produces images with size 1624 pixels by 1234 pixels.

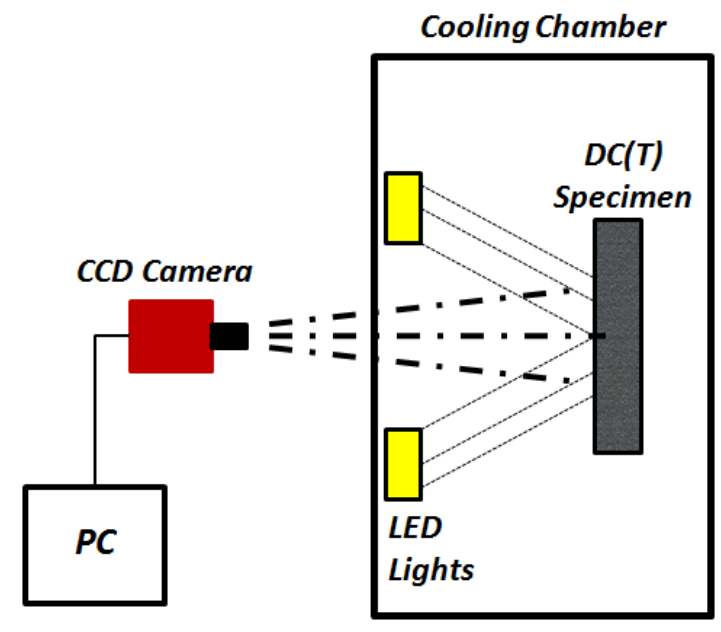

Fig. 3. DIC Schematic. 
Specimen DIC image capturing occurred at a perpendicular distance of $1 \mathrm{~m}$ from the specimen surface to the camera body. Furthermore, the region of interest was $45 \mathrm{~mm}$ in length by $45 \mathrm{~mm}$ in height. A qualitative representation of the region of interest (ROI) for the samples is shown in Fig. 4. Images were centered vertically at the notch tip and the image origin is at the upper left corner of each image with the $y$-axis increasing in a downward direction and the $x$-axis increasing in a rightward direction. All specimens were imaged prior to application of the speckle pattern to identify aggregate and mastic phases on the specimen surface.
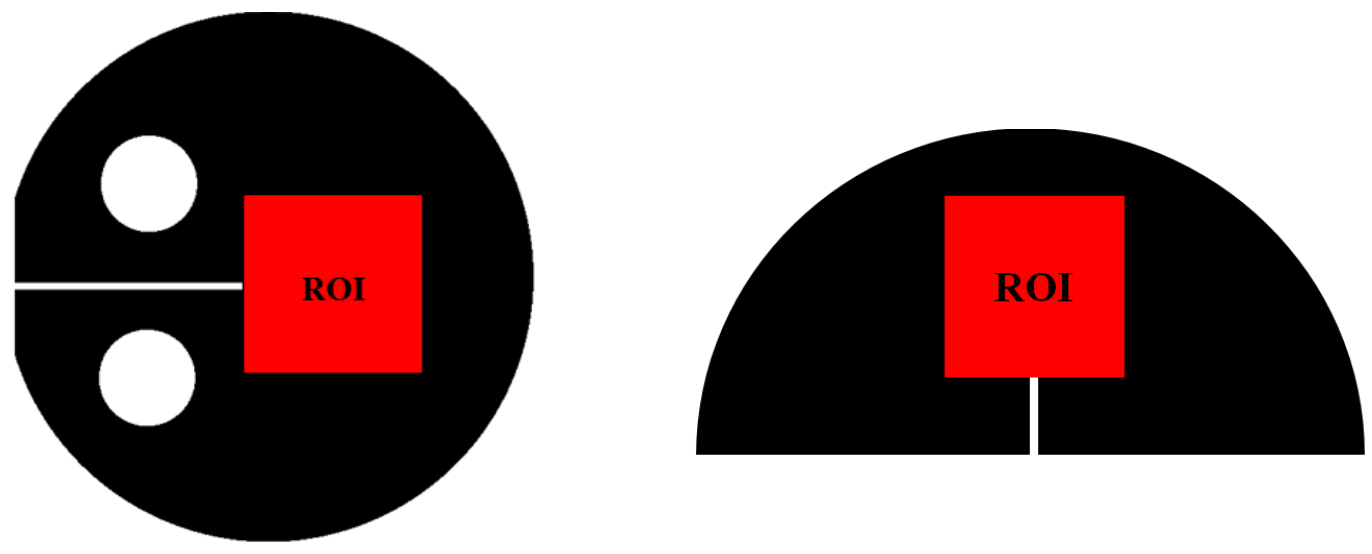

Fig. 4. Region of interest for: (a) DC(T) geometry; and (b) SCB geometry (45mm by $45 \mathrm{~mm}$ ).

The current study developed a DIC post-processing Matlab tool to measure the average size of the FPZ and the rate of FPZ change. The program imports the DIC-measured strain field data and evaluates the crack tip location in the image and FPZ. In order to evaluate these locations, it was hypothesized that locations with tensile strain measures less than $100 \mu \varepsilon$ were not part of the fracture process and locations with strain higher than $2,000 \mu \varepsilon$ were considered to be completely cracked with no tensile load carrying capacity. The $100 \mu \varepsilon$ minimum threshold was based on the study by Airey et al. (2002) which found that asphalt behaved linearly at this strain level. The crack tip locating feature finds the furthest location from the notch tip with more than $2,000 \mu \varepsilon$ as shown by the black line below in Fig. 5. The size of the FPZ is evaluated by identifying the furthest location from the crack tip with a tensile strain between 100 and $2000 \mu \varepsilon$ for the entire DIC strain field. Finally, in order to determine the rate of FPZ change, a linear trendline was fitted to the FPZ data from the peak FPZ to the final data point FPZ ( $\triangle F P Z_{\text {post max }}$ ) versus testing time $(\Delta t)$. Consequently, the slope of that line was considered the rate of FPZ change or $\triangle F P Z$ Size Slope.

$$
\triangle F P Z \text { Size Slope }=\frac{\Delta F P Z_{\text {post } \max }}{\Delta t}
$$




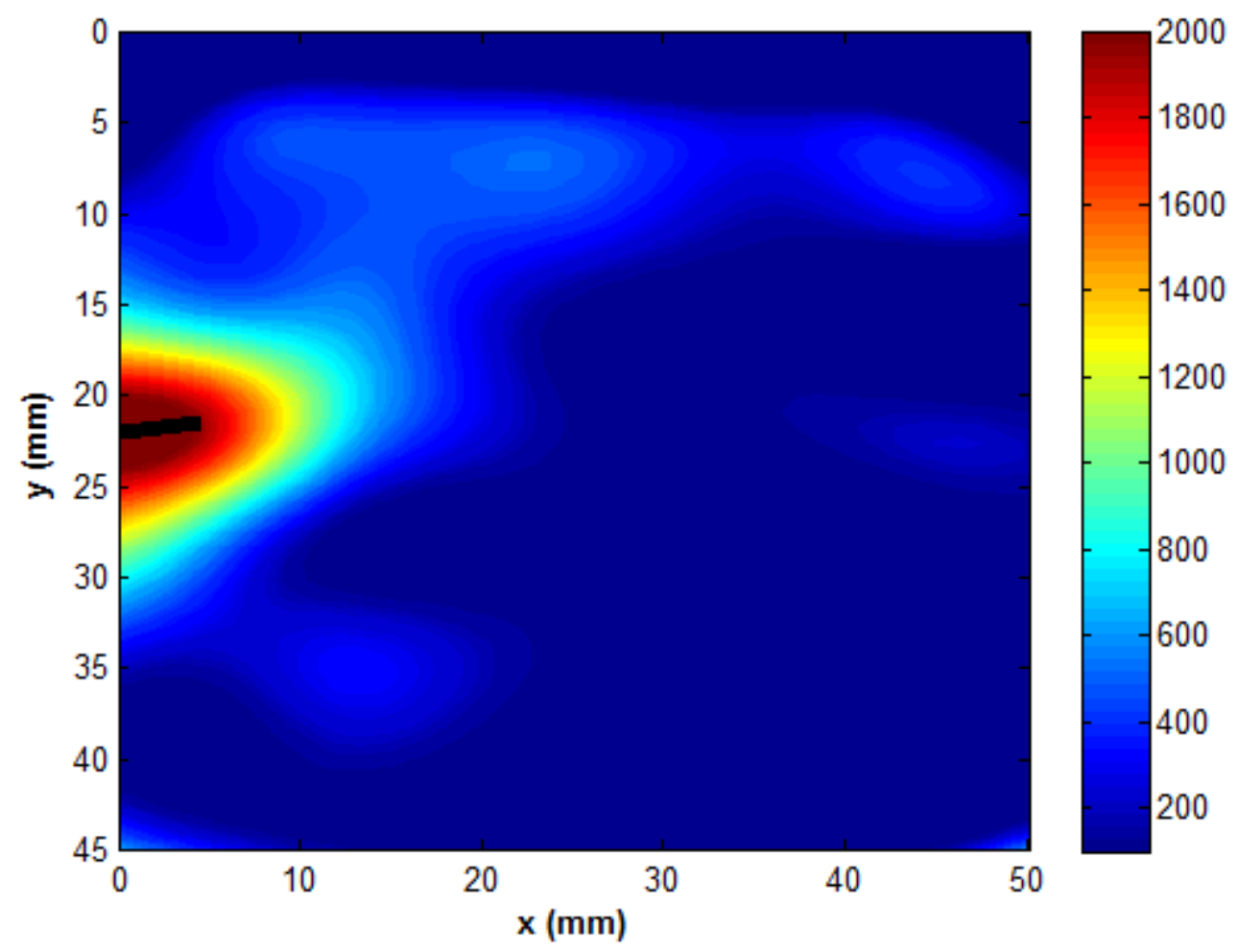

Fig. 5. PG 70-28 Fracture Process Zone and Crack Tip Location (Black line represents average crack trajectory).

\section{Results and Discussion}

\subsection{Macroscale Mixture Results}

Macroscale properties of the asphalt mixtures at high and low temperatures demonstrates a benefits of SBS polymer addition. Hamburg wheel tracking results demonstrate the stiffening effect of SBS polymer addition at high test temperatures $\left(50^{\circ} \mathrm{C}\right)$. As such, rutting resistance follows the high temperature grade of the asphalt binder and thus polymer content. The results in Fig. 6 demonstrate that the PG 70-28 mixture did not perform as well as the PG 70-22 mixture after approximately 5,000 wheel passes. This result likely occurred due to the softer low temperature grade of the material, resulting in greater creep relaxation in the PG 70-28 mixture. The change in unmodified binder from the control PG 64-22 mixture to the PG 58-28 mixture had a larger impact on the rutting resistance as compared to the use of PG 70-22 or PG 70-28 binders. This finding will be explored further in the Hamburg-DC(T) diagram. Finally, the PG 76-22 mixture did not reach the tertiary flow stage in the Hamburg test. Thus, 3.0\% SBS polymer addition significantly affected mixture rutting resistance properties in conjunction with alleviating concerns regarding moisture damage sensitivity. 


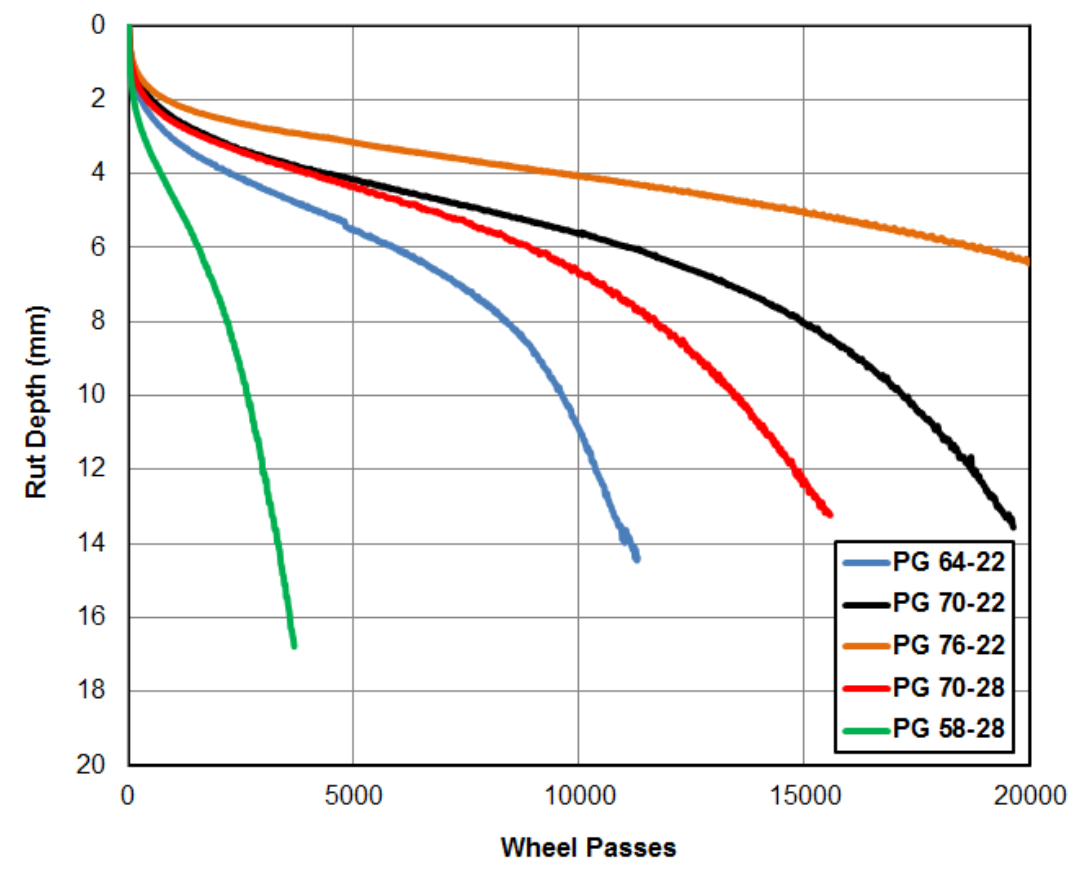

Fig. 6. Hamburg Rutting Resistance Results $\left(50^{\circ} \mathrm{C}\right)$.

The low temperature macroscale fracture properties similarly demonstrate the importance of polymer modification in both $\mathrm{DC}(\mathrm{T})$ and SCB geometries. Both polymer addition and low temperature grade of the asphalt binder affected the measured CMOD fracture energies of the asphalt mixtures in this study. In particular, the use of $-28^{\circ} \mathrm{C}$ grade asphalt binder led to the highest fracture energies in the $\mathrm{DC}(\mathrm{T})$ test with the PG 58-28 mixture yielding the highest fracture resistance among all mixtures. Similar to the Hamburg results, the largest impact in changing binder grade occurred with changes in unmodified asphalt. Further discussion will be presented below in the Hamburg-DC(T) diagram discussion. Fracture resistance in both test geometries increased in the presence of SBS polymer considering the PG 64-22 mixture as the control group. Specifically, the improvement in fracture energy in the PG 70-22 and PG 76-22 mixtures appear to be associated with the increased peak load of the material response. This improvement is hypothesized to have occurred due to the ability of the polymer additive to disperse the area of localized strain resulting in a larger FPZ without reaching complete material separation. The SCB results differed from the $\mathrm{DC}(\mathrm{T})$ results with respect to the ranking of PG 76-22 and PG 70-28 mixtures. This result likely occurred due to the bending fracture geometry and the higher impact of stiffness on that geometry in proportion to the impact of stress relaxation on the material response in the SCB. 
Table 2. DC(T) and SCB CMOD Fracture Energy Results $\left(-12^{\circ} \mathrm{C}\right)$

\begin{tabular}{|c|c|c|}
\hline \multicolumn{3}{|c|}{ DC(T) Results } \\
\hline Binder Grade & Avg. Peak Load (kN) & Avg. CMOD Gf $\left(J^{\prime} / \mathbf{m}^{2}\right)$ \\
\hline PG 64-22 & 3.209 & 551 \\
\hline PG 58-28 & 2.592 & 848 \\
\hline PG 70-22 & 3.209 & 585 \\
\hline PG 70-28 & 3.291 & 679 \\
\hline PG 76-22 & 3.586 & 615 \\
\hline \multicolumn{2}{|c|}{} & SCB Results \\
\hline PG 64-22 & 4.679 & 1055 \\
\hline PG 70-22 & 5.201 & 1064 \\
\hline PG 70-28 & 4.680 & 1117 \\
\hline PG 76-22 & 5.364 & 1290 \\
\hline
\end{tabular}

The Hamburg-DC(T) diagram demonstrates the applicability of this graphical tool to help visualize shifts in asphalt performance due to polymer addition and to develop more optimized mixture designs. As stated previously, the ultimate goal is to shift mixture behavior toward the upper-right region, i.e., perpendicular to the orientation of the binder trade-off axis thus increasing both rutting and fracture resistance. The results provided in Fig. 7 show that the length of the vector along the binder trade-off axis between the unmodified binders was longer than the shifts caused by polymer modification graphically. As such, moving from unacceptable to marginally acceptable performance does not require additional binder modifiers, and thus can be done with minimal economic investment in some cases. However, in order to shift to the more challenging goal of acceptable performance at the highest level of traffic requires more significant investment, as movement in the Hamburg-DC(T) space to the upper-right corner of the plot requires more expensive, polymer-modified binders. The PG 70-28 mixture shifted in the most perpendicular direction with respect to the binder trade-off axis by increasing significantly in both rutting and low temperature cracking resistance. This result likely manifested itself due to improved low temperature stress relaxation and high temperature stiffness properties compared to the PG 64-22 mixture. It is of interest to note that the PG 70-22 and PG 76-22 mixtures linearly shifted from the PG 64-22 mixture, but in a more vertical, thus less favorable, fashion compared to the PG 70-28 mixture. This linear shift among those three data points is hypothesized to have occurred due to the similar low temperature relaxation properties in the presence of increasing stiffness with increased polymer content. 


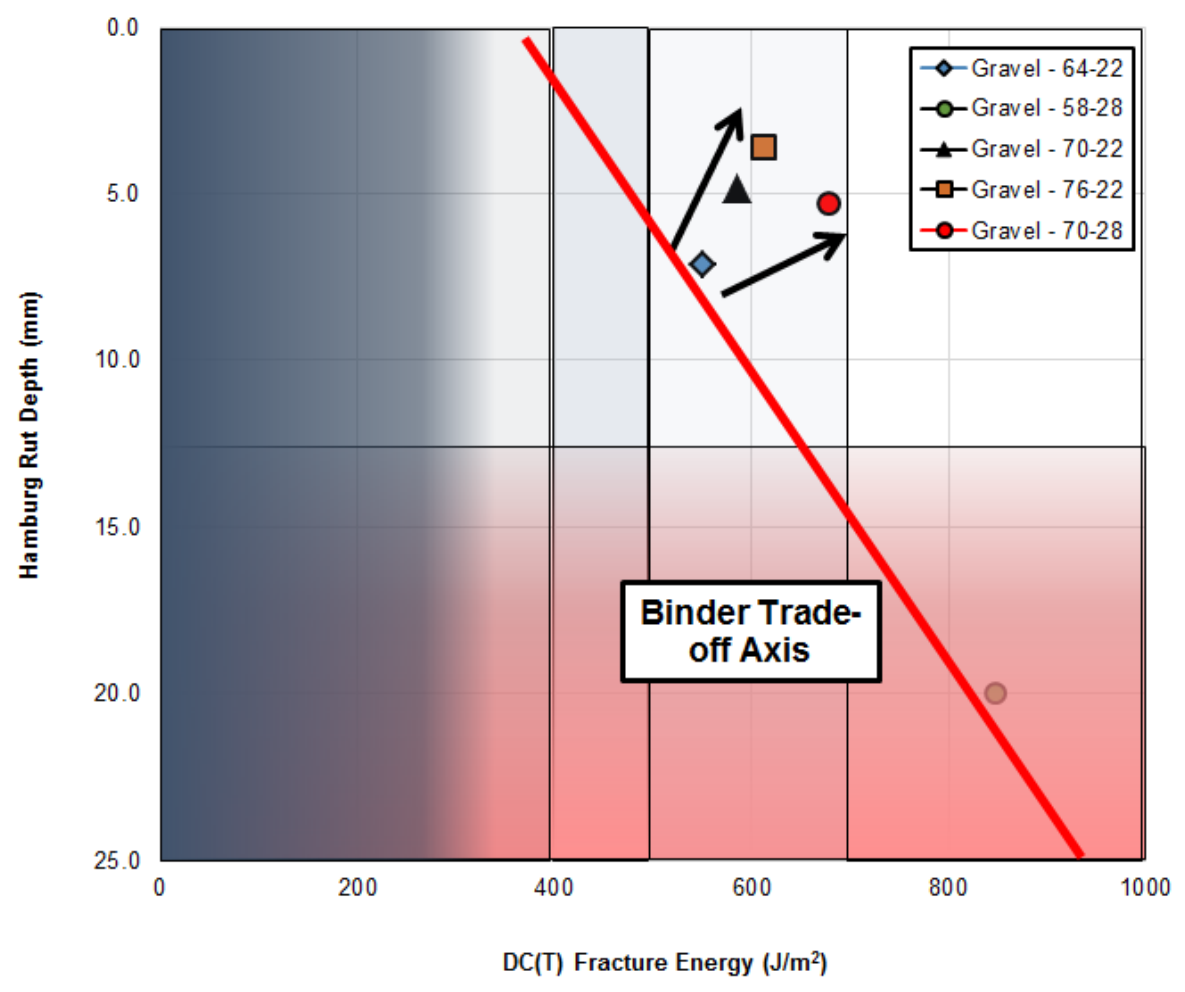

Fig. 7. Hamburg-DC(T) Diagram.

Polymer addition had a larger effect on movement toward the upper right corner of the Hamburg-SCB diagram than low temperature grade. The addition of 1.5\% SBS in the PG 70-22 and PG 70-28 led to approximately similar fracture and rutting resistance as shown in Fig. 8. The use of 3.0\% SBS in the PG 76-22 yielded the best performance results among all mixtures. The differences between the Hamburg-DC(T) and Hamburg-SCB diagrams were caused by the smaller ligament length and larger zone of compression in the SCB test as compared to the DC(T) test. As stated previously, the geometry effects likely reduced the importance of relaxation properties in contrast with the mixture stiffness. 


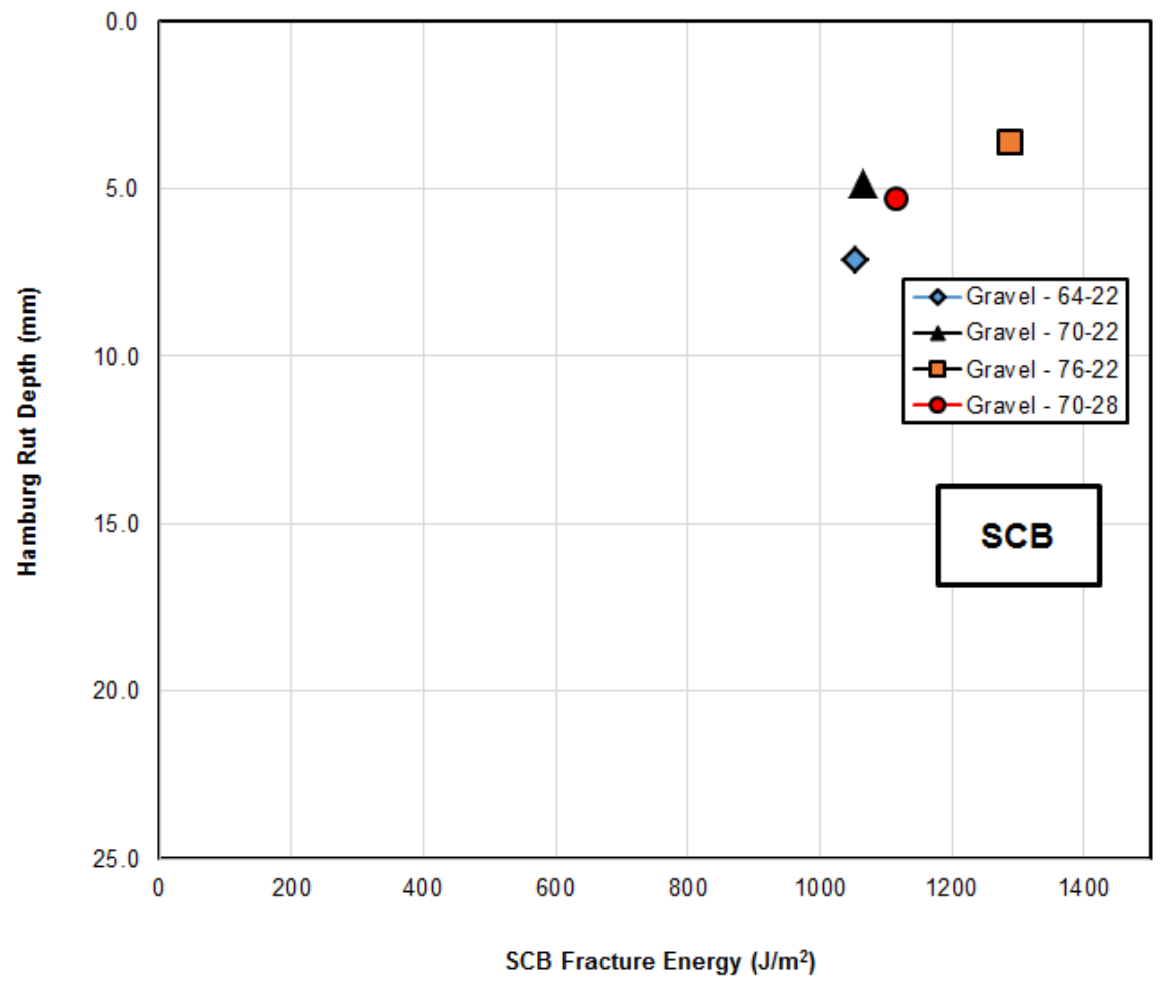

Fig. 8. Hamburg-SCB Diagram.

\subsection{Microscale Mixture Results}

The microscale mixture properties at low temperatures demonstrate the effect of polymer addition in terms of mixture crack propagation and the size and rate of change of the FPZ. The DC(T) and SCB crack movement results are shown in Fig. 9 and Fig. 10. The DC(T) test results rank in order of polymer content with PG 76-22 best and PG 64-22 worst. Thus, larger contents of polymer provided greater localized strain dispersion. The addition of $1.5 \%$ SBS polymer in addition to a softer low temperature grade, thus greater relaxation properties at $-12^{\circ} \mathrm{C}$, in the PG 70-28 mixture led to an offset in crack propagation as the crack did not begin to move until 0.04 $\mathrm{mm}$ of CMOD opening after the PG 64-22 and 70-22 mixtures. The PG 76-22 mixture with $3.0 \%$ SBS polymer provided the greatest offset between loading and the onset of crack propagation. The SCB crack movement followed the same trends as the $\mathrm{DC}(\mathrm{T})$ at longer loading times (CMOD values). In addition, there was less data spread at early loading times for the SCB geometry as opposed to the $\mathrm{DC}(\mathrm{T})$ geometry which likely occurred due to the smaller SCB ligament length. 


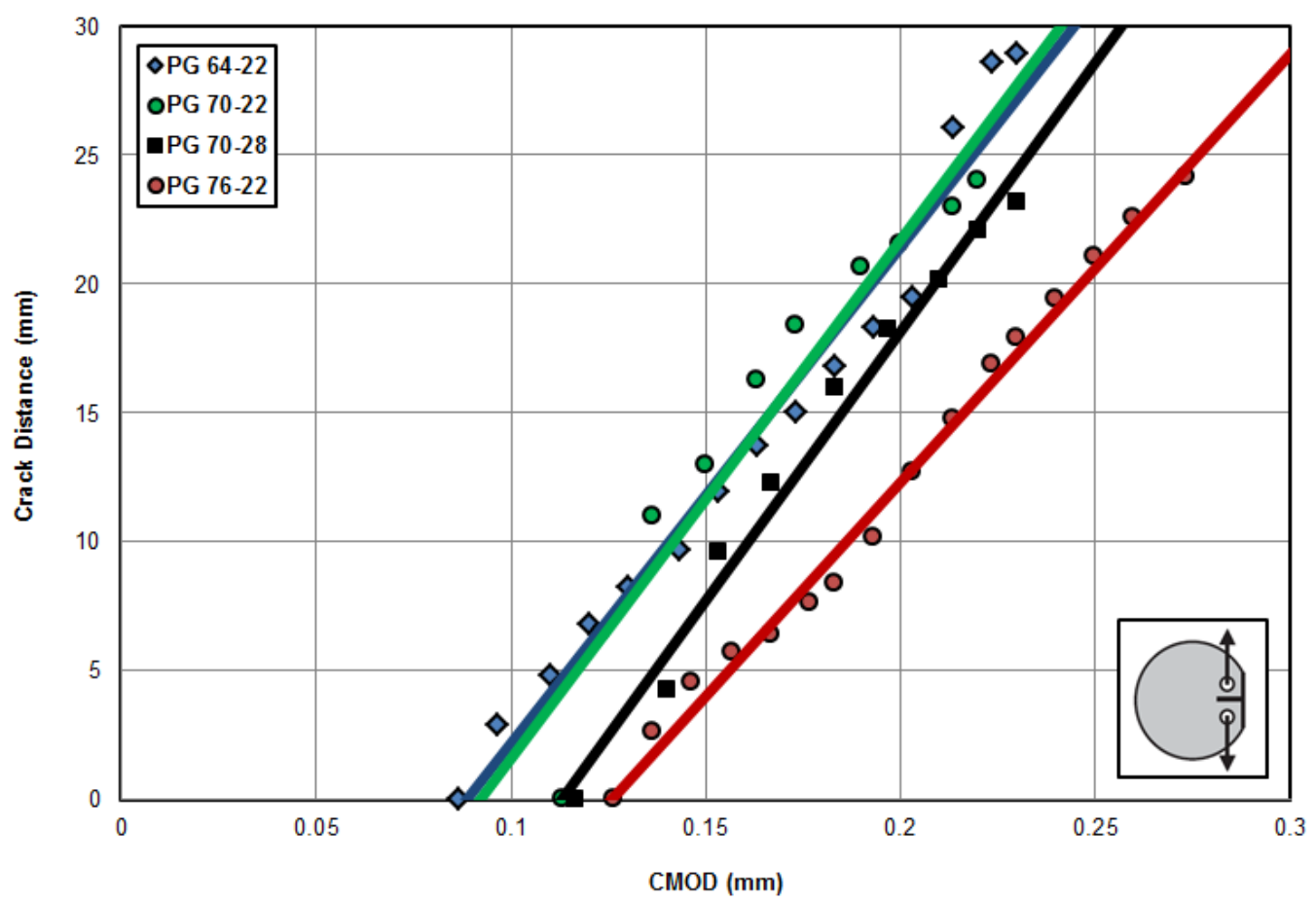

Fig. 9. Mixture Crack Propagation vs. CMOD in the DC(T) Test.

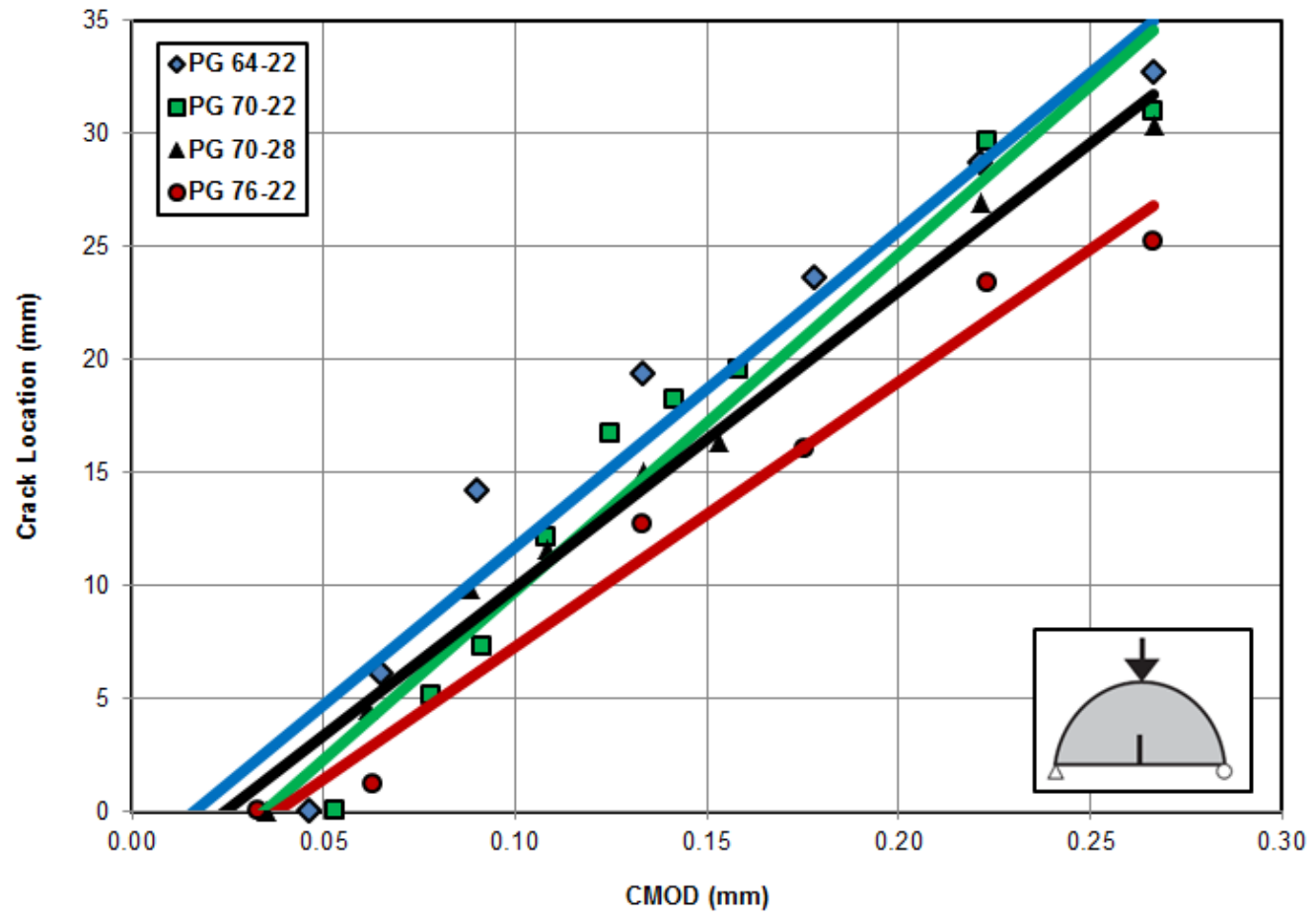

Fig. 10. Mixture Crack Propagation vs. CMOD in the SCB Test. 
The DIC measurements of FPZ and its rate of change demonstrate the effect of both geometry and polymer content. As shown below in Fig. 11 and Fig. 12, the FPZ reduces in size with the onset of crack propagation through the fracture ligament of the test specimen. This finding was anticipated due to the boundary conditions of the specimen affecting the fracture process and has been shown in numerical simulations of asphalt concrete fracture. The current study evaluated the rate of FPZ change via the $\triangle F P Z$ Size Slope. In the DC(T) geometry results, polymer content increased the size and reduced the rate of change of the FPZ as hypothesized in the previous sections of this paper. The addition of polymer dispersed the area of strain localization (FPZ) more than the unmodified asphalt binder mixture which indicated better relaxation properties and thus better low temperature cracking resistance. Furthermore, the PG 70-28 mixture performed best in terms of $\triangle F P Z$ Size Slope. The combined effect of improved binder relaxation properties, only PG XX-28 mixture evaluated in DIC, and polymer modification yielded a reduced rate of FPZ change and thus better low temperature performance at the micro- and macro-scales.

SCB FPZ results did not correlate well with the DC(T) results in terms of the effects of polymer content. Polymer modification reduced the $\triangle F P Z$ Size Slope in PG 70-28 and PG 76-22 mixtures. However, the primary finding from the SCB geometry was the reduction in FPZ size as compared to the $\mathrm{DC}(\mathrm{T})$ geometry. The reduced ligament length, boundary conditions, and large compressive zone likely caused this reduction in FPZ size.

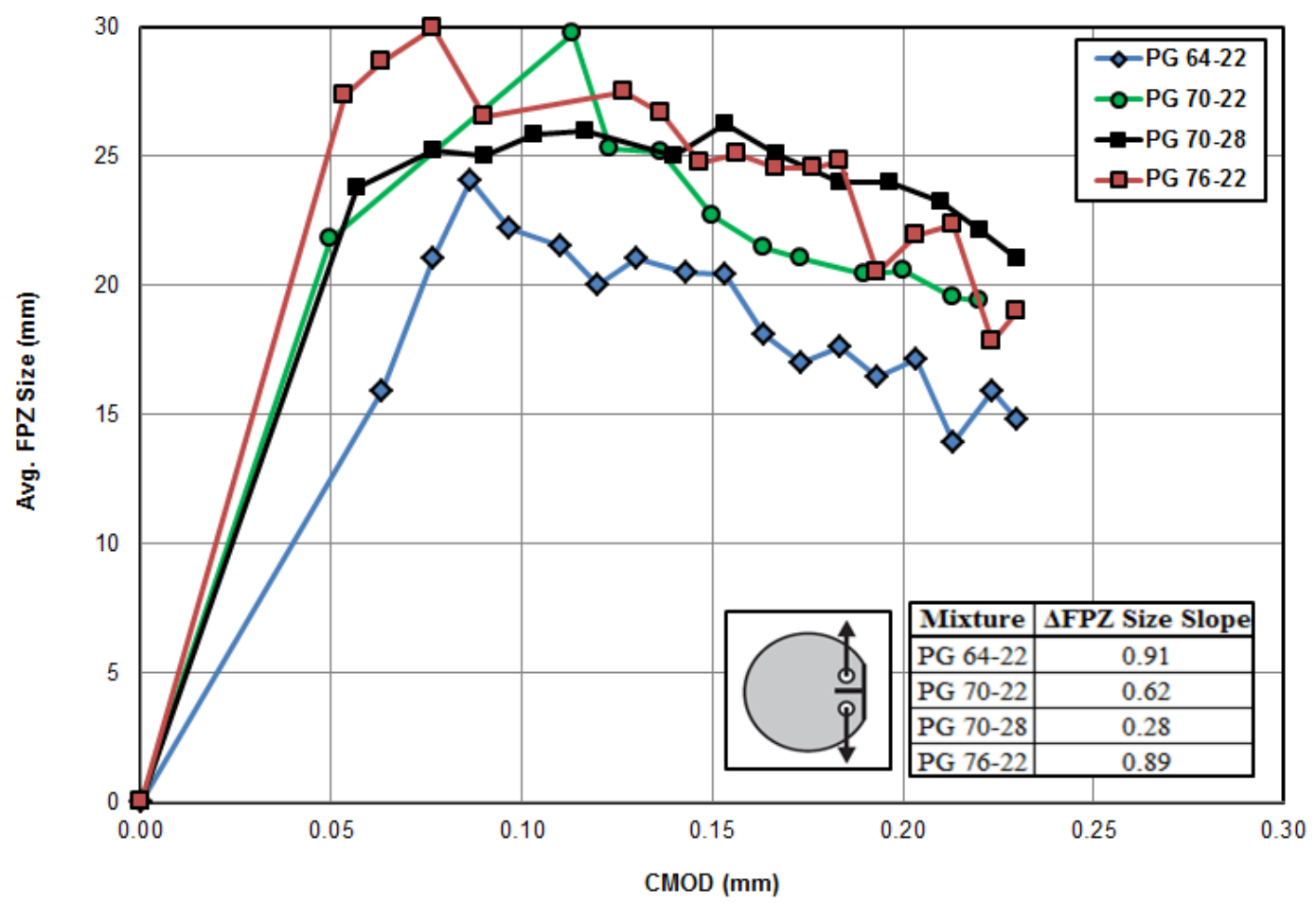

Fig. 11. Mixture FPZ Size vs. CMOD in the DC(T) Test. 


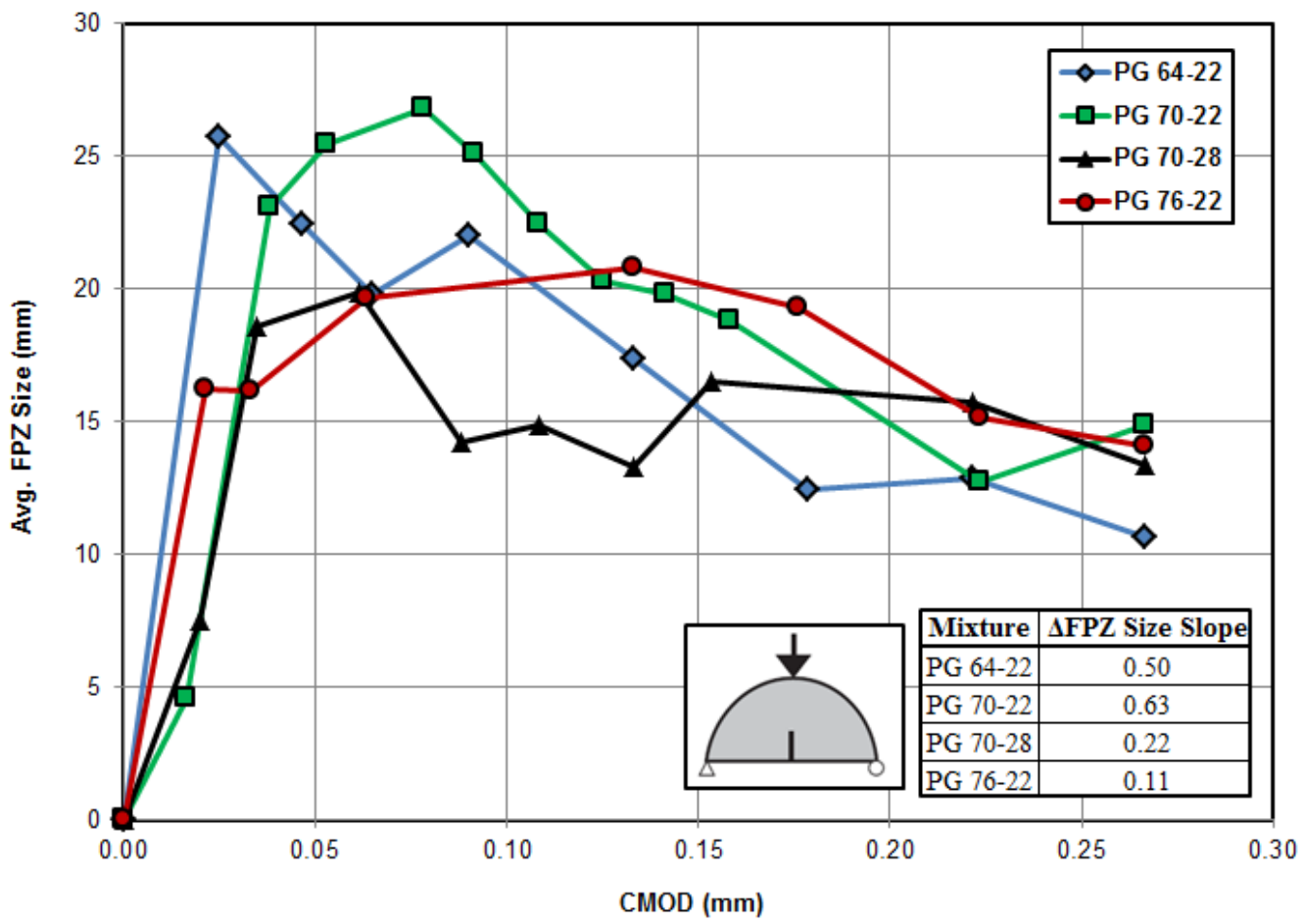

Fig. 12. Mixture FPZ Size vs. CMOD in the SCB Test.

\section{Summary and Conclusions}

The current study evaluated the effects of polymer modification in asphalt mixtures at high and low temperatures via micro- and macro-scale properties. High temperature and low temperature testing was completed using the Hamburg and DC(T) and SCB tests, respectively. The macroscale properties were evaluated in each individual test and performance interaction plots. Furthermore, microscale properties were measured using DIC camera technology at low temperatures for both $\mathrm{DC}(\mathrm{T})$ and SCB geometries. The following conclusions were drawn based on the study:

1. Both high and low temperature binder grade adjustment through the use of polymer modification can lead to high-performance mixtures. Results shown using the HamburgDC(T) diagram show that the PG 70-28 mixture yielded the best shift toward the optimum upper-right zone of the diagram, demonstrating improved high and low temperature performance as compared to the PG 64-22 mixture.

2. The FPZ of asphalt materials changes with time as it moves toward the specimen boundaries and zones of compression created in low temperature fracture tests. This phenomenon has been shown via numerical modeling approaches, but this study found it experimentally for both unmodified and modified asphalt mixtures.

3. Polymer modification generally increases the size of the FPZ with reference to the DC(T) results in this study demonstrating enhanced low temperature performance at the microscale. This greater dispersion shows that the material has a greater capacity to relax stresses such as those developed via mechanical or thermal loads. 


\section{Acknowledgement}

This material is based upon work supported by the National Science Foundation under Grant No. 1031218. Any opinions, findings, and conclusions or recommendations expressed in this material are those of the author(s) and do not necessarily reflect the views of the National Science Foundation.

\section{References}

1. Von Quintus, H., Mallela, J. (2005) Reducing Flexible Pavement Distress in Colorado Through the Use of PMA Mixtures. Colorado Asphalt Pavement Association Final Report 16729.1/1.

2. Von Quintus, H., Mallela, J., Buncher, M. (2007) Quantification of Effect of PolymerModified Asphalt on Flexible Pavement Performance. Journal of the Transportation Research Record, Vol. 2001, pp. 141-154.

3. Timm, D.H., Robbins, M.M., Willis, J.R., Tran, N., Taylor, A.J. (2013) Field and Laboratory Study of High-Polymer Mixtures at the NCAT Test Track: Final Report. National Center for Asphalt Technology Final Report 13-03, pp. 1-52.

4. Woo, W.J., Ofori-Abebresse, E., Chowdhury, A., Hibrich, J., Kraus, Z., Martin, A.E., Glover, C.J. (2007) Polymer Modified Asphalt Durability in Pavements. Texas Department of Transportation Final Report FHWA/TX-07/0-4688-1, pp. 1-424.

5. Vahidi, S., Mogawer, W.S., Booshehrian, A. (2014) Effects of GTR and Treated GTR on Asphalt Binder and High-RAP Mixtures. Journal of Materials in Civil Engineering, Vol. 26, No. 4, pp. 721-727.

6. Yan, Y., Cocconcelli, C., Roque, R., Nash, T., Zou, J., Hernando, D., Lopp, G. (2015) Performance Evaluation of Alternative Polymer-Modified Asphalt Binders. Road Materials and Pavement Design, 16:Supp. 1, pp. 389-403.

7. Mohammad, L.N., Cooper Jr., S.B., Elseifi, M.A. (2011) Characterization of HMA Mixtures Containing High Reclaimed Asphalt Pavement Content with Crumb Rubber Additives. Journal of Materials in Civil Engineering, Vol. 23, No. 11, pp. 1560-1568.

8. Birgisson, B., Montepara, A., Romeo, E., Tebaldi, G. (2011) Characterisation of Asphalt Mixture Cracking Behaviour using the Three-point Bending Beam Test. International Journal of Pavement Engineering, 12:6, pp. 569-578.

9. Montepara, A., Romeo, E., Isola, M., Tebaldi, G. (2011) The Role of Fillers on Cracking Behavior of Mastics and Asphalt Mixtures. Journal of the Association of Asphalt Paving Technologists, Vol. 80, pp. 161-192.

10. Montepara, A., Romeo, E., Birgisson, B., Tebaldi, G. (2010) Strain Localization and Damage Distribution in SBS Polymer Modified Asphalt Mixtures. Road Materials and Pavement Design, 11:4, pp. 899-915.

11. Elseifi, M.A., Mohammad, L.N., Ying, H., Cooper III, S. (2012) Modeling and Evaluation of the Cracking Resistance of Asphalt Mixtures using the Semicircular Bending Test at Intermediate Temperatures. Road Materials and Pavement Design, 13: Supp 1, pp. 124-139.

12. Braham, A., Mudford, C. (2013) Development of Fracture Resistance Curves for Asphalt Concrete. Journal of Materials in Civil Engineering, Vol. 25, No. 11, pp. 1631-1637. 
13. Zhou, F., Hu, S., Scullion, T. (2013) Balanced RAP/RAS Mix Design and Performance Evaluation System for Project-Specific Service Condition. Texas Department of Transportation Final Report FHWA/TX-13/0-6092-3, pp. 1-74.

14. Cooper III, S.B., Mohammad, L.N., Kabir, S., King Jr., W. (2014) Balanced Asphalt Mixture Design Through Specification Modification: Louisiana's Experience. Journal of the Transportation Research Record, Vol. 2447, pp. 92-100.

15. Ozer, H., Al-Qadi, I.L., Singhvi, P., Khan, T., Rivera-Perez, J., El-Katib, A. (2016) Fracture Characterization of Asphalt Mixtures with RAP and RAS using the Illinois Semicircular Bending Test Method and Flexibility Index. Accepted for presentation at the 2016 Transportation Research Board Conference, Washington, D.C.

16. Buttlar, W.G., Hill, B.C., Wang, H., Mogawer, W.S. (2016) Performance-Space Diagram for the Evaluation of High and Low Temperature Asphalt Mixture Performance. Accepted for Publication in the Association of Asphalt Paving Technologists.

17. Bazant, Z.P., Kazemi, M.T. (1990) Determination of Fracture Energy, Process Zone Length, and Brittleness Number from Size Effect, with Application to Rock and Concrete. International Journal of Fracture, Vol. 44, pp. 111-131.

18. Das, S., Kizilkanat, A., Neithalath, N. (2015) Crack Propagation and Strain Localization in Metallic Particulate-Reinforced Cementitious Mortars. Journal of Materials and Design, Vol. 79, pp. 15-25.

19. AASHTO T-324 (2014) Hamburg Wheel-Track Testing of Compacted Hot Mix Asphalt. American Association of State Highway and Transportation Officials.

20. ASTM D-7313 (2013) Standard Test Method for Determining Fracture Energy of Asphalt-Aggregate Mixtures Using the Disk-Shaped Compact Tension Geometry. American Society for Testing and Materials.

21. Roberts, F.L., Kandhal, P.S., Brown, E.R., Lee, D.Y., and Kennedy, T.W. Hot Mix Asphalt Materials, Mixture Design, and Construction. National Asphalt Pavement Association $2^{\text {nd }}$ Edition. Lanham, MD, 1996.

22. Abanto-Bueno J, Lambros J (2002) Investigation of crack growth in functionally graded materials using digital image correlation. J. Engineering Fracture Mechanics, Vol. 69, pp. 1695-1711.

23. Carroll JD, Abuzaid W, Lambros J, Sehitoglu H (2013) High resolution digital image correlation measurements of strain accumulation in fatigue crack growth. International Journal of Fatigue Vol. 57, pp. 140-150.

24. Poissant J, Barthelat F (2010) A Novel Subset Splitting Procedure for Digital Image Correlation on Discontinuous Displacement Fields. Experimental Mechanics, Vol. 50 (3), pp. 353-364.

25. Airey, G.D., Rahimzadeh, B., Collop, A. (2002) Evaluation of the Linear and Non-linear Viscoelastic Behavior of Bituminous Binders and Asphalt Mixtures. 6th International Conference on the Bearing Capacity of Roads, Railways and Airfields, Lisbon, Portugal. 